\title{
ON THE TOTAL CURVATURES OF A TAME FUNCTION
}

\author{
VINCENT GRANDJEAN
}

\begin{abstract}
Given a definable function $f: \mathbb{R}^{n} \mapsto \mathbb{R}$, enough differentiable, we study the continuity of the total curvature function $t \rightarrow K(t)$, total curvature of the level $f^{-1}(t)$, and the total absolute curvature function $t \rightarrow|K|(t)$, total absolute curvature of the level $f^{-1}(t)$. We show they admits at most finitely many discontinuities.
\end{abstract}

\section{INTRODUCTION}

One of the nicest feature of o-minimal structures expanding the ordered field of real numbers is that taking the derivative of a definable function (in a given such o-minimal structure) provides a definable function (in the same given o-minimal structure). Unfortunately the inverse operation is somehow much more delicate, integration and measure lead to problems even in some of their simplest aspects.

In the setting of subanalytic geometry some measure theoretical aspects, density, Lipschitz-killing curvatures, of the subanalytic sets had been studied [BB, Com, $[\mathrm{Fu}]$. Still in this context, the $k$-dimensional volume of a global subanalytic subset of $\mathbb{R}^{n}$, lying in a globally subanalytic family of subsets of $\mathbb{R}^{n}$ of dimension at most $k$, is, when finite, a log-analytic function in the parameter, as proved in [LR, CLR]. This is already an issue since the logarithmic contribution cannot be avoided and so the functions carrying the quantitative aspect of the variation of the volume in the parameter of the family are already outside of the structure.

In the world of non-polynomially bounded o-minimal structures expanding the real numbers, almost nothing similar to the statement in the globally subanalytic context is known in whole generality. Let us nevertheless mention the results of [Le, Ka], proved independently, namely the definability of the set of the parameters at which the 2-dimensional volume of a definable family of plane definable subsets is finite.

2000 Mathematics Subject Classification. Primary 03C64, 53B20 ; Secondary 49Q15, $57 \mathrm{R} 35$.

Key words and phrases. o-minimal structure, Gauss Curvature, Hausdorff Limits, Morse index.

Partially supported by the European research network IHP-RAAG contract number HPRN-CT-2001-00271. 
Let us go closer to the goal of this note. Given a definable function $f: \mathbb{R}^{n} \mapsto \mathbb{R}$, that is $C^{l}$ with $l \geqslant 2$, to each regular level $t$ we associate two real numbers, namely, $K(t)$, the total curvature of the level $f^{-1}(t)$ oriented by the unitary gradient field of $f$, and, $|K|(t)$, the total absolute curvature of the level $f^{-1}(t)$. When $n$ is odd and $f^{-1}(t)$ is compact and connected, the Gauss-Bonnet-Chern Theorem states that $K(t)$ is just the Euler Characteristic of $f^{-1}(t)$ (modulo a constant depending only on $n$ ). Somehow $K(t)$ and $|K|(t)$ have a connection with the topological equisingularity type of $f^{-1}(t)$, even when $f^{-1}(t)$ is no longer compact. Thus knowing the variation as a function of $t$ of these total curvatures functions could give some information about the equisingularity of the family of the levels. This was the first motivation of this study (see $[\mathrm{Gr}]$ for some results in this direction).

From the measure theoretical point of view, these total curvatures are just weighted $n$-1-dimensional volumes of a definable 1-parameter family of subsets of $\mathbb{S}^{n-1}$. So how do the total curvature functions in the parameter behave? As already said, for the level of generality we want to deal with, there is no hope (yet!) to provide some quantitative information about the variation of these functions of $t$. Nevertheless we propose in this note to study some qualitative properties of the functions $t \rightarrow K(t)$ and $t \rightarrow|K|(t)$, and we will find some. We actually proved

Theorem. Let $f: \mathbb{R}^{n} \mapsto \mathbb{R}$ be a $C^{l}, l \geqslant 2$, definable function.

(1) the function $t \rightarrow|K|(t)$ admits at most finitely many discontinuities.

(2) If the function $t \rightarrow|K|(t)$ is continuous at a regular value $c$ of $f$, so is the function $t \rightarrow K(t)$.

The paper is organized as follows.

In Section 2 we provide some definitions, conventions and notations that will be used in the rest of the note.

Section 3 recalls what the total curvature and the total absolute curvature of a hypersurface are and what are the connections with linear orthogonal projections onto oriented lines.

In Section 4 we just define the Gauss map of a given function and states some of its elementary properties in the frame established in Section 3 .

Section 5 is devoted to Hausdorff limits of Gauss images since they will be the key tool of our main result.

Theorem 6.4 and Proposition 6.6 are the main results of Section 6, and are proved with the help of some preliminary work.

In Section 7 we state results of the same flavor as those of Section 6 , but for what we named the total $\lambda$-curvature and total absolute $\lambda$-curvature.

We finish this paper with some remarks in Section 8 . 


\section{NOTATION - CONVENTION}

Let $\mathbb{R}^{n}$ be the real $n$-dimensional affine space endowed with its Euclidean metric. The scalar product will be denoted by $\langle\cdot, \cdot\rangle$.

Let $\mathbb{B}_{R}^{n}$ be the open ball of $\mathbb{R}^{n}$ centered at the origin and of radius $R>0$.

Let $\mathbb{S}_{R}^{n-1}$ be the $(n-1)$-sphere centered at the origin and of radius $R>0$.

Let $\mathbb{S}^{n-1}$ be unit ball of $\mathbb{R}^{n}$ endowed with the induced Euclidean metric and let $d_{\mathbb{S}}$ be the intrinsic distance function on $\mathbb{S}^{n-1}$.

Let $\mathrm{d} v_{k}$ be the $k$-dimensional Hausdorff measure of $\mathbb{R}^{n}$ with $k \in\{1, \ldots, n\}$.

Let us recall briefly what an o-minimal structure is.

An $o$-minimal structure $\mathcal{M}$ expanding the ordered field of real numbers is a collection $\left(\mathcal{M}_{p}\right)_{p \in \mathbb{N}}$, where $\mathcal{M}_{p}$ is a set of subsets of $\mathbb{R}^{p}$ satisfying the following axioms

1) For each $p \in \mathbb{N}, \mathcal{M}_{p}$ is a boolean sub-algebra of subsets of $\mathbb{R}^{p}$.

2) If $A \in \mathcal{M}_{p}$ and $B \in \mathcal{M}_{q}$, then $A \times B \in \mathcal{M}_{p+q}$.

3) If $\pi: \mathbb{R}^{p+1} \mapsto \mathbb{R}^{p}$, is the projection on the first $p$ factors, given any $A \in$ $\mathcal{M}_{p+1}, \pi(A) \in \mathcal{M}_{p}$.

4) The algebraic subsets of $\mathbb{R}^{p}$ belongs to $\mathcal{M}_{p}$.

5) $\mathcal{M}_{1}$ consists exactly of the finite unions of points and intervals.

So the smallest o-minimal structure is the structure of the semi-algebraic subsets.

Assume that such an o-minimal structure $\mathcal{M}$ is given for the rest of this article.

A subset $A$ of $\mathbb{R}^{p}$ is a definable subset (in the given o-minimal structure) of $\mathbb{R}^{p}$, if $A \in \mathcal{M}_{p}$.

A mapping $g: X \mapsto \mathbb{R}^{q}$, where $X \subset \mathbb{R}^{p}$, is a definable mapping (or just definable, for short) if its graph is a definable subset of $\mathbb{R}^{p+q}$.

The reader may refer to [Cos, vD1, vDM] to learn more on the properties of definable subsets and definable mappings.

let $Z$ be a connected definable subset of $\mathbb{R}^{n}$. The dimension of $Z, \operatorname{dim} Z$, is well defined.

A point $z_{0} \in Z$ is smooth if there exists a neighborhood $U \subset \mathbb{R}^{n}$ of $z_{0}$, such that $U \cap Z$ is diffeomorphic to $\mathbb{R}^{k}$, for an integer $k \leqslant \operatorname{dim} Z$. The property of being smooth of a given dimension is a locally open property once $Z$ is equipped with the induced topology.

A point $z_{0}$ which is not smooth is called singular. The set of such points is definable. 
Let $S_{1}$ and $S_{2}$ be respectively $C^{1}$ definable submanifolds of $\mathbb{R}^{n}$ and of $\mathbb{R}^{p}$. Let $g: S_{1} \mapsto S_{2}$ be a $C^{1}$ definable function. A point $x_{0}$ is a smooth or regular point of $g$ if the rank $d_{x_{0}} g$ is maximum in a neighborhood of $x_{0}$.

A critical or singular point $x_{0}$ of $g$ is a point at which $d_{x_{0}} g$ is not of maximum rank. The set of such points is definable, denoted by $\operatorname{crit}(g)$.

By abuse of language, we will talk about the rank of the mapping $g$ at a point $x_{0}$ to mean the rank of the differential $\mathrm{d}_{x_{0}} g$.

A definable family $\mathcal{E}=\left(E_{t}\right)_{t \in T}$ of subsets of a definable submanifold $S \subset \mathbb{R}^{n}$, with parameter space $T \subset \mathbb{R}^{m}$ does not only mean that $E_{t}$ is a definable subset of $S$, but that the subset $\left\{(x, t) \in S \times T: x \in E_{t}\right\}$ is definable. Equivalently it means it is the family of the (projection onto $S$ of the) fibers of a definable mapping.

\section{TOTAl CURVATURE AND TOTAL ABSOlute CURVATURE OF A CONNECTED ORIENTABLE HYPERSURFACE}

Let $M$ be a definable connected, $C^{2}$ hypersurface of $\mathbb{R}^{n}$. Assume $M$ is orientable and the orientation is given by a $C^{1} \operatorname{map}, M \ni x \rightarrow v_{M}(x) \in \mathbb{S}^{n-1}$. The map $v_{M}$ is definable.

Let $\mathcal{U} \subset \mathbb{S}^{n-1}$ be defined as $\boldsymbol{v}_{M}\left(M \backslash \operatorname{crit}\left(\mathrm{v}_{M}\right)\right)$, where $\operatorname{crit}\left(\mathrm{v}_{M}\right)$ is the set of critical points of $v_{M}$. The subset $\mathcal{U}$ is a definable open subset and, when not empty, for any $u \in \mathcal{U}, v_{M}^{-1}(u)$ is finite. By Gabrielov uniformity theorem, there exists a positive integer $N_{M}$, such that $\# v_{M}^{-1}(u) \leqslant N_{M}$ for any $u \in \mathcal{U}$.

Since $\mathcal{U}$ is a finite disjoint union of open definable subsets $U_{i}, i=1, \ldots, d$, let $M_{i}=v_{M}^{-1}\left(U_{i}\right) \backslash \operatorname{crit}\left(v_{M}\right)$ which is a definable subset of $M$. Let $s(i)$ be the number of points in a fiber above any $u \in U_{i}$.

Let $k_{M}(x)$ be the Gauss curvature at $x \in M$, that is the value of the determinant of the Jacobian matrix of $v_{M}$. We make the convention that the $(n-1)$-dimensional volume of the empty set is 0 .

Proposition 3.1. The total absolute curvature of $M$ is

$$
|K|_{M}:=\int_{M}\left|k_{M}(x)\right| \mathrm{d} v_{n-1}(x)=\sum_{i} s(i) \operatorname{vol}_{n-1}\left(U_{i}\right) .
$$

Proof. If the maximal rank of $\mathrm{v}_{M}$ is at most $n-2$, then $\operatorname{crit}\left(\mathrm{v}_{M}\right)=M$ and so we deduce $|K|_{M}=0$.

So assume that $\mathrm{v}_{M}$ is at most $n-1$.

Let us denote by Jac, the Jacobian of any mapping (when it makes sense). Since the mapping $v_{M}$ is of maximal rank, the set of its critical values of 
$\mathrm{v}_{M}$, that is $\mathrm{v}_{M}\left(\operatorname{crit}\left(\mathrm{v}_{M}\right)\right)$, is of codimension at least 1 in $\mathbb{S}^{n-1}$. Each $U_{i}$ is open and so is each $M_{i}$ in $M$.

We assume first that each connected component of $M_{i}$ is simply connected. Then $M_{i}=M_{i}^{(1)} \sqcup \ldots \sqcup M_{i}^{s(i)}$ and $v_{M}$ induces a diffeomorphism from $M_{i}^{(j)}$ onto $U_{i}$, for each $j=1, \ldots, s(i)$. Thus we find

$$
\operatorname{vol}_{n-1}\left(U_{i}\right)=\int_{U_{i}} \mathrm{~d} v_{n-1}=\int_{M_{i}^{(j)}} \mathbf{J a c}\left(v_{M}(x)\right) \mathrm{d} v_{n-1}(x), \text { for each } j .
$$

Thus we deduce

$$
\int_{M_{i}} \mathbf{J a c}\left(v_{M}(x)\right) \mathrm{d} v_{n-1}(x)=s(i) \operatorname{vol}_{n-1}\left(U_{i}\right) .
$$

As another consequence of this fact, if we define $M^{*}$ as $M \backslash\left(\sqcup_{i} M_{i}\right)$, then $M^{*}$ is definable, and since $v_{M}\left(M^{*}\right)$ is at most of dimension $n-2$ (meaning that $\operatorname{Jac}\left(\mathrm{v}_{M}\right)$ is zero on a definable dense open set of $M^{*}$ if $\left.\operatorname{dim} M^{*}=n-1\right)$, we obtain

$$
\int_{M^{*}} \mathbf{J a c}\left(v_{M}(x)\right) \mathrm{d} v_{n-1}(x)=0 .
$$

Then

$$
|K|_{M}=\int_{M} \mathbf{J a c}\left(\mathbf{v}_{M}(x)\right) \mathrm{d} v_{n-1}(x)=\sum_{i} \int_{M_{i}} \mathbf{J a c}\left(v_{M}(x)\right) \mathrm{d} v_{n-1}(x),
$$

which is the desired result.

In the general situation, by the cylindrical decomposition theorem, there exists a closed subset $N_{i}$ of $M_{i}$ of dimension at most $n-2$, such that each connected component of $M_{i} \backslash N_{i}$ is simply connected. Then we do the same as above with $v_{M}\left(M_{i} \backslash N_{i}\right)$ instead of $U_{i}$. Since $\operatorname{vol}_{n-1}\left(v_{M}\left(N_{i}\right)\right)=0$, the formula given is still true in this general case

For each $i=1, \ldots, m$, let $\sigma_{i}^{+}$be $\#\left(v_{M}^{-1}(u) \cap\left\{\operatorname{det} k_{M}>0\right\}\right)$ and $\sigma_{i}^{-}$be $\#\left(v_{M}^{-1}(u) \cap\left\{\operatorname{det} k_{M}<0\right\}\right)$, for any $u \in U_{i}$, since these numbers depend only on $U_{i}$. We deduce that $s(i)=\sigma_{i}^{+}+\sigma_{i}^{-}$and thus

$$
|K|_{M}=\sum_{i}^{N_{M}}\left(\sigma_{i}^{+}+\sigma_{i}^{-}\right) \operatorname{vol}_{n-1}\left(U_{i}\right) .
$$

Let $\sigma_{i}=\sigma_{i}^{+}-\sigma_{i}^{-}$. Note that $\sigma_{i}=\operatorname{deg}_{u} v_{M}=: \operatorname{deg}\left(\left.v_{M}\right|_{v_{M}^{-1}\left(U_{i}\right)}\right)$ the degree of the mapping $v_{M}$ at any $u \in U_{i}$.

Proposition 3.2. The total curvature of $M$ is

$$
K_{M}:=\int_{M} k_{M}(x) \mathrm{d} v_{n-1}(x)=\sum_{i=1}^{N_{M}} \sigma_{i} \operatorname{vol}_{n-1}\left(U_{i}\right) .
$$


Proof. It works exactly as in the proof of Proposition 3.1 .

Let us come to a more specific property of the Gauss map. For any $u \in$ $\mathbb{S}^{n-1}$, let $\varphi_{u}(x):=\langle x, u\rangle$ be the orthogonal projection on the oriented vector line $\mathbb{R} u$.

Let us consider the following

Lemma 3.3. Let $y \in M$ be a point at which the rank of $\mathrm{d}_{y} v_{M}$ is $n-1$. Let $u=v_{M}(y)$. Then the function $\left.\varphi_{u}\right|_{M}$ has a Morse singular point at $y$, that is the Hessian matrix $\operatorname{Hess}_{y}\left(\left.\varphi_{u}\right|_{M}\right)$ is non degenerate.

Proof. Let us identify the hyperplane $T_{y} M$ with $\mathbb{R}^{n-1}$ with coordinates $\mathbf{x}=$ $\left(x_{1}, \ldots, x_{n-1}\right)$ centered at $y$ and remember that $\mathrm{d}_{y} \mathrm{v}_{M}$ can be seen as a reflexive endomorphism of $T_{y} M=T_{u} \mathbb{S}^{n-1}$. Since it is of rank $n-1$, it has exactly $(n-1)$ non-zero real eigenvalues.

By the definable implicit function theorem, there exist an open neighborhood $\mathscr{U}$ of the origin in the hyperplane $T_{y} M$ and a $C^{1}$-definable map $\phi: \mathscr{U} \mapsto \mathbb{R}$ such that there exists a neighborhood $\mathscr{V}$ of $y$ in $M$ such that $\mathscr{V}=\left\{x_{n}=\varphi(\mathbf{x}): \mathbf{x} \in \mathscr{U}\right\}$. Thus we find that $\left.\varphi_{u}\right|_{M}(x)=\phi(\mathbf{x})$. We thus rewrite $\mathrm{v}_{M}$ as

$$
v_{M}(x)=v_{M}(\mathbf{x})=\frac{\nabla\left(x_{n}-\phi(\mathbf{x})\right)}{\left|\nabla\left(x_{n}-\phi(\mathbf{x})\right)\right|} .
$$

For any $u \in T_{y} M$ we find that

$$
\begin{aligned}
\mathrm{d}_{y} \overline{\mathbf{v}}_{M} \cdot \xi & =\frac{1}{\left|\nabla\left(x_{n}-\phi(\mathbf{x})\right)\right|}\left[\operatorname{Hess}_{y} \phi \cdot \xi-\left\langle\operatorname{Hess}_{y} \phi \cdot \xi, u\right\rangle u\right] \\
& =\frac{1}{\left|\nabla\left(x_{n}-\phi(\mathbf{x})\right)\right|} \operatorname{Hess}_{y} \phi \cdot \xi,
\end{aligned}
$$

where $\operatorname{Hess}_{y} \phi$ is the Hessian matrix of $\phi$ at $y$. Since $\mathrm{d}_{y} \mathrm{v}_{M}$ is of rank $n-1$, we deduce that $\operatorname{Hess}_{y}\left(\left.\varphi_{u}\right|_{M}\right)=\operatorname{Hess}_{y} \phi$, and thus is Morse at $y$.

Thanks to Lemma 3.3 we can then define the following

Definition 3.4. The Morse index of $M$ at $x \notin \operatorname{crit}\left(v_{M}\right)$ is the integer $\lambda_{M}(x)$ define as the Morse index at $x$ of the function $\left.\varphi_{u}\right|_{M}$, with $u=v_{M}(x)$.

Obviously this definition depends on the choice of $v_{M}$.

Let $U$ be a connected component of $\mathcal{U}=\mathrm{v}_{M}(M) \backslash \mathrm{v}_{M}\left(\operatorname{crit}\left(\mathrm{v}_{M}\right)\right)$. Thus $v_{M}$ induces a finite $C^{l-1}$ covering

$$
\mathrm{v}_{M}: M_{1} \sqcup \ldots \sqcup M_{d} \mapsto U .
$$

Proposition 3.5. With the previous notation, the function $M_{j} \ni x \rightarrow \lambda_{M}(x)$ is definable and so is constant. 
Proof. Each subset $M_{j}$ is a definable and open in $M$. Given an orthonormal basis of $\mathbb{R}^{n}$, let $P(x ; T) \in \mathbb{R}[T]$ be the characteristic polynomial of $\operatorname{Hess}_{x}\left(\varphi_{v_{M}(x)} \mid M\right)$. The coefficients of this polynomial are definable functions of $x \in M \backslash \operatorname{crit}\left(v_{M}\right)$ and $C^{l-1}$. As functions of $x$, the roots (counted with multiplicity) are continuous and definable and all non zero. Since $M_{j}$ is connected, the number of negative roots is constant and so is the index $\lambda_{M}(x)$.

There is a straightforward corollary of Lemma 3.3 and Proposition 3.5

Corollary 3.6. Let $y \in M$ be a point at which the rank of $\mathrm{d}_{y} v_{M}$ is $n-1$. Let $u=v_{M}(y)$. Then the function Morse index of $\left.\varphi_{u}\right|_{M}$ and the index of $\mathrm{d}_{y} \mathrm{v}_{M}$ are the same.

Thus we can also rewrite the total curvature as

$$
K_{M}=\int_{v_{M}(M) \backslash v_{M}\left(\operatorname{crit}\left(v_{M}\right)\right)}\left(\sum_{x \in v_{M}^{-1}(u) \backslash \operatorname{crit}\left(v_{M}\right)}(-1)^{\lambda_{M}(x)}\right) \mathrm{d} v(u),
$$

since the set of points $v_{M}^{-1}\left(\operatorname{crit}\left(v_{M}\right)\right) \cap\left(M \backslash \operatorname{crit}\left(v_{M}\right)\right)$ is at most of dimension $n-2$.

\section{ON THE GAUSS MAP OF A TAME FUNCTION}

Let $f: \mathbb{R}^{n} \mapsto \mathbb{R}$, be a definable function, enough differentiable, say $C^{l}$, with $l \geqslant 2$.

Let us denote by $K_{0}(f)$ the set of critical values of $f$.

For each $t$, let $F_{t}$ be the level $f^{-1}(t)$.

The Gauss map of the function $f$ is the following mapping

$$
\begin{array}{cl}
v_{f}: \mathbb{R}^{n} \backslash \operatorname{crit}(f) & \mapsto \mathbb{S}^{n-1} \\
x & \rightarrow \frac{\nabla f(x)}{|\nabla f(x)|}
\end{array}
$$

It is a definable and $C^{l-1}$ mapping. We will denote by $v_{t}$ the restriction $\left.v_{f}\right|_{F_{t}}$, providing an orientation to each (connected component of the) level $F_{t}$ which is compatible with the transverse structure of the foliation (on $\left.\mathbb{R}^{n} \backslash \mathbf{c r i t}(f)\right)$ by the connected components of the (regular) levels of $f$. Note that $\operatorname{crit}\left(\mathrm{v}_{f}\right) \cap F_{t}=\operatorname{crit}\left(\mathrm{v}_{t}\right)$.

The differential mapping of $v_{f}$ at $x \notin \operatorname{crit}(f)$, is

$$
\mathrm{d}_{x} \mathrm{v}_{f}: \xi \mapsto \frac{1}{|\nabla f(x)|}\left[\operatorname{Hess}_{x}(f) \cdot \xi-\left\langle\operatorname{Hess}_{x}(f) \cdot \xi, v_{f}(x)\right\rangle \boldsymbol{v}_{f}(x)\right]
$$

We recall that for any $x \in F_{t}$, the linear mapping $\mathrm{d}_{x} v_{t}=\left.\mathrm{d}_{x} \mathrm{v}_{f}\right|_{T_{x} F_{t}}$ seen as an endomorphism of $T_{v_{f}(x)} \mathbb{S}^{n-1}$ is reflexive. 
Definition 4.1. Let $x \notin \operatorname{crit}(f) \cup \operatorname{crit}\left(v_{f}\right)$. The tangent Gauss index of $f$ at $x$, denoted by $\lambda_{f}(x)$ is the index of the reflexive endomorphism $\left.\mathrm{d}_{x} \mathrm{v}_{f}\right|_{T_{x} f^{-1}(x)}$.

Proposition 4.2. The function $x \rightarrow \lambda_{f}(x)$ is a locally constant definable mapping.

Proof. Assume a fixed orthonormal basis of $\mathbb{R}^{n}$ is given. Let $P(x, T) \in \mathbb{R}[T]$ be the characteristic polynomial of the endomorphism $\left.\mathrm{d}_{x} v_{f}\right|_{T_{x} f^{-1}(x)}$. From the computation of the differential $\mathrm{d}_{x} v_{f}$ we deduce that the coefficients of this polynomial are definable and $C^{l-2}$. Given $x$, let $\alpha_{1}(x) \leqslant \ldots \leqslant \alpha_{n-1}(x)$ be the roots of this polynomial. For each $i=1, \ldots, n-1$, the functions $x \rightarrow \alpha_{i}(x)$ is continuous and definable. For this reason the number of negative roots (that is the tangent Gauss index) is constant on each connected components of $\mathbb{R}^{n} \backslash\left(\operatorname{crit}(f) \cup \operatorname{crit}\left(v_{f}\right)\right)$, that is $x \rightarrow \lambda_{f}(x)$ is constant on $\mathbb{R}^{n} \backslash\left(\operatorname{crit}(f) \cup \operatorname{crit}\left(\mathrm{v}_{f}\right)\right)$.

Since the mapping $x \rightarrow \lambda_{f}(x)$ is constant on each connected component of $\mathbb{R}^{n} \backslash\left(\operatorname{crit}(f) \cup \operatorname{crit}\left(\mathrm{v}_{f}\right)\right)$, then its graph is a definable subset of $\mathbb{R}^{n} \times \mathbb{R}$ since $\mathbb{R}^{n} \backslash\left(\operatorname{crit}(f) \cup \operatorname{crit}\left(\mathrm{v}_{f}\right)\right)$ is a definable subset of $\mathbb{R}^{n}$.

This property will be very useful in Section 6 .

When $\operatorname{crit}\left(\mathrm{v}_{f}\right) \neq \emptyset$, let us consider the following $C^{l-1}$ definable mapping: $\Psi_{f}: \mathbb{R}^{n} \backslash\left(\operatorname{crit}(f) \cup \operatorname{crit}\left(\mathrm{v}_{f}\right)\right) \mapsto \mathbb{S}^{n-1} \times \mathbb{R}$ defined as $x \rightarrow\left(\mathrm{v}_{f}(x), f(x)\right)$.

This mapping is definable and $C^{l-1}$. It is also a local diffeomorphism at any of the point of $\mathbb{R}^{n} \backslash\left(\operatorname{crit}(f) \cup \operatorname{crit}\left(v_{f}\right)\right)$, thus its image $\widetilde{\mathcal{U}}$ is open.

Definition 4.3. Let $(u, t) \in \widetilde{\mathcal{U}}$. The tangent Gauss degree of $f$ at $(u, t)$, denoted $\operatorname{deg}_{f}(u, t)$ is the degree of $\Psi_{f}$ at $(u, t)$.

Then we get the following,

Proposition 4.4. The function $(u, t) \rightarrow \operatorname{deg}_{f}(u, t)$ is a locally constant definable mapping.

Proof. This comes from the fact that $\Psi_{f}$ is a local diffeomorphism at each point of its definition domain.

More interestingly we also have

Proposition 4.5. For any $(u, t) \in \widetilde{\mathcal{U}}$,

$$
\operatorname{deg}_{(u, t)} \Psi_{f}=\operatorname{deg}_{u} v_{t}=\sum_{x \in v_{t}^{-1}(u)}(-1)^{\lambda_{f}(x)} .
$$

Proof. Let $y \in \Psi_{f}^{-1}(u, c)$. Let $\mathscr{F}:=\left(v_{1}, \ldots, v_{n-1}, v_{n}\right)$, with $v_{n}=v_{f}$, be a $C^{1}$ and direct orthonormal frame in a neighborhood $\mathcal{Y}$ of $y$. With such 
"coordinates", for any $x \in F_{t} \cap \mathcal{Y}$, both $\mathrm{d}_{x} \Psi_{f}$ and $\mathrm{d}_{x} \nu_{t}$ are considered as endomorphisms. For $x \in F_{t} \cap \mathcal{Y}$, writing the matrices of $\mathrm{d}_{x} \Psi_{f}$ and $\mathrm{d}_{x} v_{t}$ in this frame, gives

$$
\operatorname{det}\left(\operatorname{Mat}_{\mathscr{F}(x)} \mathrm{d}_{x} \Psi_{f}\right) \cdot \operatorname{det}\left(\operatorname{Mat}_{\mathscr{F}(x)} \mathrm{d}_{x} \mathrm{v}_{t}\right)>0,
$$

since the frame respects the orientations of $\mathbb{R}^{n}$ and of $T_{x} F_{t}$ and where Mat $\mathscr{F}(x)$ denotes the matrix in the base $\mathscr{F}(x):=\left(v_{1}(x), \ldots, v_{n-1}(x), v_{n}(x)\right)$. Thus the signs of these determinants are the same and so is proved the lemma.

\section{HAUSDORFF LIMITS OF GAUSS IMAGES}

We use notations of Section 4

We recall that $\widetilde{\mathcal{U}}=\Psi_{f}\left(\mathbb{R}^{n} \backslash\left(\operatorname{crit}(f) \cup \operatorname{crit}\left(v_{f}\right)\right)\right)$ is open and definable in $\mathbb{S}^{n-1} \times \mathbb{R}$ and that for any $(u, t) \in \widetilde{\mathcal{U}}, \Psi_{f}^{-1}(u, t)$ is finite.

From Gabrielov Uniformity theorem, there exists a positive integer $N_{f}$ such that for any $(u, t) \in \widetilde{\mathcal{U}}, \# \Psi_{f}^{-1}(u, t) \leqslant N_{f}$.

Let us define the following definable sets

$$
\begin{aligned}
& \widetilde{\mathcal{U}}_{k}=\left\{(u, t) \in \widetilde{\mathcal{U}}: \# \Psi_{f}^{-1}(u, t)=k\right\} . \\
& \mathcal{U}_{t}=\left\{u \in \mathbb{S}^{n-1}:(u, t) \in \widetilde{\mathcal{U}}\right\}=v_{t}\left(F_{t} \backslash \operatorname{crit}\left(v_{f}\right)\right)=v_{t}\left(F_{t} \backslash \operatorname{crit}\left(v_{t}\right)\right) . \\
& \mathcal{U}_{k, t}=\left\{u \in \mathbb{S}^{n-1}:(u, t) \in \widetilde{\mathcal{U}}_{k}\right\} .
\end{aligned}
$$

The subsets $\mathcal{U}_{t}$ and $\mathcal{U}_{k, t}$ are open in $\mathbb{S}^{n-1}$.

Note that at any point of $\mathbb{R}^{n} \backslash\left(\operatorname{crit}(f) \cup \operatorname{crit}\left(\boldsymbol{v}_{f}\right)\right), \Psi_{f}$ is a local diffeomorphism.

Let us remark that both families $\left(\mathcal{U}_{t}\right)_{t \in f\left(\mathbb{R}^{n}\right) \backslash K_{0}(f)}$ and $\left(\mathcal{U}_{k, t}\right)_{t \in f\left(\mathbb{R}^{n}\right) \backslash K_{0}(f)}$ are definable families of open subsets of $\mathbb{S}^{n-1}$.

Let $\mathcal{K}\left(\mathbb{R}^{n}\right)$ be the space of compact subsets of $\mathbb{R}^{n}$.

Given $Y$ and $Z$ compacts subsets of $\mathbb{R}^{n}$, the Hausdorff distance between $Y$ and $Z$, denoted by $\mathrm{d}_{\mathcal{K}\left(\mathbb{R}^{n}\right)}(Y, Z)$, is defined as

$$
\begin{aligned}
& \mathrm{d}_{\mathcal{K}\left(\mathbb{R}^{n}\right)}(Y, Z)=\max \left(\min _{y \in Y} \operatorname{dist}(y, Z), \min _{z \in Z} \operatorname{dist}(z, Y)\right) \\
&=\min \{r \geqslant 0: \forall y \in Y \text { and } \forall z \in Z, \operatorname{dist}(y, Z) \leqslant r \\
&\text { and } \operatorname{dist}(z, Y) \leqslant r\}
\end{aligned}
$$

The space $\mathcal{K}\left(\mathbb{R}^{n}\right)$ equipped with the Hausdorff distance $\mathrm{d}_{\mathcal{K}\left(\mathbb{R}^{n}\right)}$ becomes a complete metric space.

Bounded definable families of compacts subsets behave well under the Hausdorff limit, (see [Br, vD2, LS] for a general frame). Since we are only interested in 1-parameter families of such subsets, the statement of the next 
result is given in this context and in the form we will use it below in the rest of this paper.

Theorem $5.1([\mathrm{Br}, \underline{\mathrm{vD} 2}, \mathrm{\textrm {LS }}])$. Let $\left(\mathcal{C}_{t}\right)_{t \in[0,1[}$ be a definable family of closed subsets of $\mathbb{R}^{n}$. Assume there exists a compact subset $Q$ such that $\mathcal{C}_{t} \subset Q$ for each $t \in\left[0,1\left[\right.\right.$. Then, the Hausdorff limit $\mathcal{C}_{1}:=\lim _{t \rightarrow 1} \mathcal{C}_{t}$ does exist and is a definable subset of $\mathbb{R}^{n}$ contained in $Q$.

Let $\left(\mathcal{C}_{t}\right)_{t \in I}$ be a definable family of closed subsets of $\mathbb{S}^{n-1}$. Since there exists a constant $C$, such that given any $u, v \in \mathbb{S}^{n-1}, \mathrm{~d}_{\mathbb{S}}(u, v) \leqslant C|u-v|$, taking Hausdorff limits of closed subsets $\left(\mathcal{C}_{t}\right)_{t \in I}$ of $\mathbb{S}^{n-1}$, will provide exactly the same Hausdorff limits of $\left(\mathcal{C}_{t}\right)_{t \in I}$ considered as closed subsets of $\mathbb{R}^{n}$.

Let us return to our topic. Given a value $c$ let us denote respectively by $\mathscr{V}_{c}^{+}$and by $\mathscr{V}_{c}^{-}$the following Hausdorff limits

$$
\mathscr{V}_{c}^{+}:=\lim _{t \rightarrow c^{+}} \operatorname{clos}\left(\mathcal{U}_{t}\right) \text { and } \mathscr{V}_{c}^{-}:=\lim _{t \rightarrow c^{-}} \operatorname{clos}\left(\mathcal{U}_{t}\right) .
$$

For each $k=1, \ldots, N_{f}$ and for $*=+,-$, let $\mathscr{V}_{k}^{*}$ be the Hausdorff limit $\lim _{t \rightarrow c^{*}} \operatorname{clos}\left(\mathcal{U}_{k, t}\right)$.

Proposition 5.2. Let $U_{1}, \ldots, U_{d_{c}}$ be the connected components of $\mathcal{U}_{c}$. For each $i=1, \ldots, d_{c}$, there exist positive integers $l_{-}=l_{-}(i) \geqslant s(i)$ and $l_{+}=$ $l_{+}(i) \geqslant s(i)$ such that $U_{i} \cap \mathscr{V}_{c}^{-} \subset \mathscr{V}_{l_{-}^{-}}^{-}$and $U_{i} \cap \mathscr{V}_{c}^{+} \subset \mathscr{V}_{l_{+}^{+}}$.

Proof. Let $u \in \mathbb{S}^{n-1}$. When not empty, the subset

$$
\Psi_{f}^{-1}(\{u\} \times \mathbb{R})=\left\{x \notin \operatorname{crit}(f) \cup \operatorname{crit}\left(v_{f}\right): v_{f}(x)=u\right\}
$$

is a $C^{l-1}$ definable curve since $\Psi_{f}$ is a local diffeomorphism. Let $\Gamma$ be a connected component of $\Psi_{f}^{-1}(\{u\})$ which intersects with $F_{c}$. Since the function $\left.f\right|_{\Gamma}$ is strictly monotonic, there exists $\varepsilon>0$ such that for any $t \in] c-\varepsilon, c[\cup] c, c+\varepsilon\left[\right.$, the curve $\Gamma$ also intersects with $F_{t}$, that is $u \in \mathcal{U}_{t}$. Since the number of connected components of $\Psi_{f}^{-1}(\{u\} \times \mathbb{R})$ meeting $F_{c}$ does depend only on $U_{i}$, the Proposition is proved.

As a consequence of Proposition 5.2 we obtain

Corollary 5.3. Let $c$ be a value such that $\mathcal{U}_{c} \neq \emptyset$. Then $\mathcal{U}_{c} \subset \mathscr{V}_{c}^{+} \cap \mathscr{V}_{c}^{-}$.

Proof. It is contained in the proof of Proposition 5.2.

The statement of Corollary 5.3 would be wrong for a general definable family $\left(\mathcal{C}_{t}\right)_{t}$ of closed subsets of $\mathbb{S}^{n-1}$. As read in the proof, the transverse structure given by the function $f$ is somehow also carried in the family $\left(\mathcal{U}_{t}\right)_{t}$, and thus explain this result. 
We end this section with an elementary result about the volume of a Hausdorff limit of a 1 parameter definable family.

Proposition 5.4. Let $\mathcal{C}:=\cup_{t \in[0,1[} \mathcal{C}_{t}$ be a definable family of closed connected subsets of dimension $n$ of $Q \in \mathbb{R}^{n}$ or $\mathbb{S}^{n}$, a compact subset. Let $\mathcal{C}_{1}$ be the Hausdorff limit $\lim _{t \rightarrow 1} \mathcal{C}_{t}$, then

$$
\lim _{t \rightarrow 1} \operatorname{vol}_{n}\left(\mathcal{C}_{t}\right)=\operatorname{vol}_{n}\left(\mathcal{C}_{1}\right) \text {. }
$$

Proof. According to [LS, Theorem 1] there exist at most finitely many subsets of $\mathcal{K}\left(\mathbb{R}^{n}\right)$ that belong to $\operatorname{clos}(\mathcal{C}) \backslash \mathcal{C} \subset \mathcal{K}\left(\mathbb{R}^{n}\right)$. By [vD2, Proposition $3,2]), C_{1}$ is well defined and definable, and its dimension is at most $n$. Note that $\mathcal{C}_{1}$ is necessarily connected.

Assume first that $Q \subset \mathbb{R}^{n}$.

Let $T_{\varepsilon}\left(\mathcal{C}_{t}\right)$ be the $\varepsilon$-neighborhood of $\mathcal{C}_{t}$ in $\mathbb{R}^{n}$ for $\varepsilon>0$.

Let $r$ be the dimension of $\mathcal{C}_{1}$. Using a generalized Weyl-Steiner's tube formula for compact definable subsets of $\mathbb{R}^{n}([\overline{\mathrm{BK}}])$, we deduce that for small $\varepsilon>0$ there exists a positive constant $L$ such that

$$
\left|\operatorname{vol}_{r}\left(C_{1}\right)-\frac{\operatorname{vol}_{n}\left(T_{\varepsilon} C_{1}\right)}{\sigma_{n-r, n} \varepsilon^{n-r}}\right|<L \varepsilon,
$$

where $\sigma_{n-r, n}$ is the volume of the unit ball $\mathbb{B}_{n-r}$ of $\mathbb{R}^{n-r}$.

By definition of $\mathcal{C}_{1}$, for any $\varepsilon>0$, there exists $\eta=\eta(\varepsilon)$ such that for $t>$ $1-\eta$ we get $\mathrm{d}_{\mathcal{K}\left(\mathbb{R}^{n}\right)}\left(\mathcal{C}_{t}, \mathcal{C}_{1}\right)<\varepsilon$, that is $\mathcal{C}_{t} \subset T_{\varepsilon}\left(\mathcal{C}_{1}\right)$.

If $r=n$, we deduce that $\operatorname{vol}_{n}\left(\mathcal{C}_{t}\right)-\operatorname{vol}_{n}\left(\mathcal{C}_{1}\right) \rightarrow 0$ as $\varepsilon \rightarrow 0$, since $\mathcal{C}_{t} \subset T_{\varepsilon}\left(C_{1}\right)$. When $r<n$ we know $\operatorname{vol}_{n}\left(T_{\varepsilon}\left(C_{1}\right)\right) \rightarrow 0$ as $\varepsilon \rightarrow 0$. So $\operatorname{vol}_{n}\left(C_{t}\right)$ must tend to 0 as $t \rightarrow 1$ (otherwise $\limsup _{t \rightarrow 1} \operatorname{vol}_{n}\left(C_{t}\right)>0$, which would contradict $\left.\lim _{\varepsilon \rightarrow 0} \operatorname{vol}_{n}\left(T_{\varepsilon}\left(\mathcal{C}_{1}\right)\right)=0\right)$.

Assume now $Q \subset \mathbb{S}^{n}$. The generalized Weyl's tube formula ([BB],[BK]) works also in spaces of constant curvatures. Thus the proof above in the flat case, adapts almost readily to the case of $\mathbb{S}^{n}$.

\section{Continuity OF THE TOTAL CURVATURE AND OF THE TOTAL ABSOLUTE CURVATURE OF A DEFINABLE FUNCTION}

We use the notations of Section 4.

Given $t \notin K_{0}(f)$, let $k_{t}$ be the Gauss curvature of $F_{t}$ with respect to the orientation $v_{t}$. Let $\mathcal{E}_{t}$ be the set of the connected components of $F_{t}$. When $t \notin K_{0}(f)$, we define the total absolute curvature of $F_{t}$ as

$$
|K|(t):=\sum_{E \in \mathcal{E}_{t}}|K|_{E},
$$


and the total curvature of $F_{t}$ as

$$
K(t):=\sum_{E \in \mathcal{F}_{t}} K_{E} .
$$

Assume $c$ is a value taken by $f$ such that $\mathcal{U}_{c}$ is not empty. Let $U_{1}, \ldots, U_{d_{c}}$, be the connected components of $\mathcal{U}_{c}$. For each $i=1, \ldots, d_{c}$, let $s(i)$ be the number of points in the fiber $v_{c}^{-1}(u)$ above any $u \in U_{i}$, that is $U_{i} \subset \mathcal{U}_{s(i), c}$, and let $\sigma_{i}(c)$ be the degree of $v_{c}$ on $U_{i}$ (see Section 3 ). We recall that

$$
\begin{gathered}
|K|(c)=\sum_{l=1}^{N_{f}} l \cdot \operatorname{vol}_{n-1}\left(\operatorname{clos}\left(\mathcal{U}_{l, c}\right)\right)=\sum_{i=1}^{d_{c}} s(i) \operatorname{vol}_{n-1}\left(\operatorname{clos}\left(U_{i}\right)\right) . \\
K(c)=\sum_{i=1}^{d_{c}} \sigma_{i}(c) \operatorname{vol}_{n-1}\left(\operatorname{clos}\left(U_{i}\right)\right)
\end{gathered}
$$

As a consequence of the result of the existence of definable Hausdorff Limits ([vD2], [LS]) and of the definition of the total curvature we deduce the following

Corollary 6.1. The following limits,

$$
\lim _{t \rightarrow c^{-}} K(t), \lim _{t \rightarrow c^{+}} K(t), \lim _{t \rightarrow c^{-}}|K|(t) \text { and } \lim _{t \rightarrow c^{+}}|K|(t)
$$

do exist, once given a value $c$.

Proof. It is just a consequence of the formulae for the total curvatures given above and of Proposition 5.4.

Let us recall that given $*=-,+$, we denote by $\mathscr{V}_{c}^{*}$, the Hausdorff limit $\lim _{t \rightarrow c^{*}} \operatorname{clos}\left(\mathcal{U}_{t}\right)$ and given any integer $1 \leqslant k \leqslant N_{f}, \mathscr{V}_{k}^{*}$ stands for the Hausdorff $\operatorname{limit}_{\lim } \rightarrow c^{*} \operatorname{clos}\left(\mathcal{U}_{k, t}\right)$

We can write $\mathscr{V}_{c}^{-} \cap \mathscr{V}_{c}^{+}$as the partition $\cup_{0 \leqslant k, l \leqslant N_{f}} \mathscr{V}_{k, l}$, where $\mathscr{V}_{k, l}=\mathscr{V}_{k}^{-} \cap$ $\mathscr{V}_{l}^{+}$, if $k, l$ are both positive, $\mathscr{V}_{k, 0}$ stands for $\mathscr{V}_{k}^{-}$if the intersection with $\mathscr{V}_{c}^{+}$is empty, $\mathscr{V}_{0, l}$ stands for $\mathscr{V}_{l}^{+}$if the intersection with $\mathscr{V}_{c}^{-}$is empty, and $\mathscr{V}_{0,0}=\emptyset$.

Our goal is still to try to understand the continuity of the functions $|K|$ and $K$. Regarding the results of Sections 3 and 5 , the non zero contribution to $|K|(t)$ only comes from the subset $\mathcal{U}_{t}$.

Proposition 6.2. Let $c$ be a value. Then

$$
|K|(c) \leqslant \min \left\{\lim _{t \rightarrow c^{-}}|K|(t), \lim _{t \rightarrow c^{+}}|K|(t)\right\} .
$$


Proof. If $\mathcal{U}_{c}$ is empty this means that $|K|(c)=0$, and so the statement is trivially true.

Let $U_{1}, \ldots, U_{d_{c}}$ be the connected components of $\mathcal{U}_{c}$. For each $i \in\left\{1, \ldots, d_{c}\right\}$ and each $*=+,-$, let $l_{*}(i)$ be the corresponding integer of Proposition 5.2 . Observe that for $*=+$ and $*=-$

$$
|K|(c)=\sum_{i} s(i) \operatorname{vol}_{n-1}\left(U_{i}\right) \leqslant \sum_{i} l_{*}(i) \operatorname{vol}_{n-1}\left(U_{i} \cap \mathscr{V}_{c}^{*}\right),
$$

and the right hand side term is $\leqslant \sum_{l} l \operatorname{vol}_{n-1}\left(\mathscr{V}_{l}^{*}\right)$. By Proposition 5.4, we conclude the proof.

The continuity of $|K|$ at $c$ implies that each of these inequalities is an equality. Using notation of Proposition 5.2 we actually obtain

Corollary 6.3. If $|K|$ is continuous at $c$, then

(1) for each pair $k, l$ with $k \neq l$, $\operatorname{vol}_{n-1}\left(\mathscr{V}_{k, l}\right)=0$, or equivalently $\mathscr{V}_{k, l}$ is of dimension at most $n-2$.

(2) for each $k$ and each $*=+,-, \operatorname{vol}_{n-1}\left(\mathscr{V}_{k}^{*}\right)=\operatorname{vol}_{n-1}\left(\mathcal{U}_{k, c}\right)$.

Proof. Using Proposition 5.2, equation 6.1 and Proposition 6.2 and writing down the continuity at $c$ provides the different statements.

Now we can come to the first main result of this section

Theorem 6.4. The function $|K|$ has at most finitely many discontinuities.

Proof. This is a consequence of Proposition 5.4 and Proposition 5.2 .

Assume $k$ is given. Let $\mathcal{C}:=\cup_{t \in f(\mathbb{R}) \backslash K_{0}(f)} \mathcal{C}_{t}$, where $\mathcal{C}_{t}=\operatorname{clos}\left(\mathcal{U}_{k, t}\right)$.

Assume first that $f(\mathbb{R}) \backslash K_{0}(f)$ is connected, so that we can assume that $\left.f(\mathbb{R}) \backslash K_{0}(f)=\right] 0,1\left[\right.$. Then $\mathcal{C}$ is a definable family of subsets of $\mathbb{S}^{n-1}$, and then is definable in $\mathcal{K}\left(\mathbb{R}^{n}\right)$. Thus its closure $\operatorname{clos}(\mathcal{C})$ in $\mathcal{K}\left(\mathbb{R}^{n}\right)$ and consists of $C$ and finitely many closed definable subsets of $\mathbb{S}^{n-1}$ by [LS, Theorem 1]. This means there exist at most finitely many $\left.c \in f(\mathbb{R}) \backslash K_{0}(f)=\right] 0,1[$

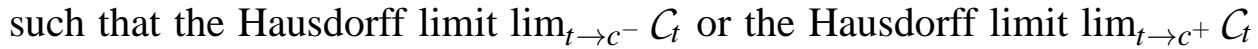
is not $\mathcal{C}_{c}$. For any value $\left.c \in\right] 0,1\left[\operatorname{such}\right.$ that $\lim _{t \rightarrow c^{-}} \mathcal{C}_{t}=\lim _{t \rightarrow c^{+}} \mathcal{C}_{t}=\mathcal{C}_{c}$, Proposition 5.4 states that $t \rightarrow \operatorname{vol}_{n-1}\left(C_{t}\right)$ is continuous at such a $c$. Since there are only finitely many $k$ and finitely many connected components of $f(\mathbb{R}) \backslash K_{0}(f)$, the theorem is proved.

Remark 6.5. Working with globally subanalytic functions only, Theorem 6.4 is then just a consequence of Lion-Rolin's Theorem [LR].

Now let us investigate the continuity of $K, t \rightarrow K(t)=\int_{F_{t}} k_{t}$.

Let us pick a value $c$. Let $\left\{U_{i}\right\}_{i=1}^{d_{c}}$ be the set of connected components of $\mathcal{U}_{c}$. We recall that $\operatorname{deg}_{u} v_{c}$, the degree of $v_{c}$ at $u \in U_{i}$ is only dependent on $i$, and is equal to $\sigma_{i}(c)$. Thus $K(c)=\sum_{i=1}^{d_{c}} \sigma_{i}(c) \operatorname{vol}_{n-1}\left(U_{i}\right)$. 
Proposition 6.6. If $|K|$ is continuous at $c$, then $K$ is continuous at $c$.

Proof. If $|K|(c)=0$, then we immediately get $K(c)=0$, and so $K$ is continuous at 0 .

Assume that $\mathcal{U}_{c}$ is not empty. Let $U_{c}$ be a connected component of $\mathcal{U}_{c}$. Then for each $u \in U$, the number of connected components of $\Psi_{f}^{-1}(\{u\} \times$ $\mathbb{R})$ meeting $F_{c}$ is constant, say equal to $l \geqslant 1$. Let $\Gamma_{1}(u), \ldots, \Gamma_{l}(u)$ be these connected components. Given $u \in \mathcal{U}_{c}$, we know that there exists $\varepsilon>0$ such that for any $t \in] c-\varepsilon, c[\cup] c, c+\varepsilon[$, we get

$$
\Psi_{f}^{-1}(\{u\} \times \mathbb{R}) \cap F_{t}=\cup_{i=1}^{l}\left(\Gamma_{i}(u) \cap F_{t}\right) .
$$

From Proposition 4.4, we know that the tangent Gauss degree of $f$, that is the degree of $v_{t}$ at $u$ is a locally constant function of $(u, t)$. Thus for $u \in \mathcal{U}_{c}$ given, there is $\varepsilon>0$ as above such that for any $t \in] c-\varepsilon, \varepsilon\left[, \operatorname{deg}_{u} v_{t}=\operatorname{deg}_{u} v_{c}\right.$. Let $\widetilde{\mathcal{V}}$ be the connected component of $\widetilde{\mathcal{U}}$ that contains $(u, c)$. Thus $\widetilde{\mathcal{V}} \cap$ $\mathbb{S}^{n-1} \times\{c\}=U_{c} \times\{c\}$ is connected. So there exists $\varepsilon>0$ such that for any $t \in] c-\varepsilon, c+\varepsilon\left[, \widetilde{\mathcal{V}} \cap \mathbb{S}^{n-1} \times\{t\}=U_{t} \times\{t\}\right.$ is connected, so $U_{t}$ is connected. Since $|K|$ is continuous, this implies that $\lim _{t \rightarrow c} \operatorname{vol}_{n-1}\left(U_{t}\right)=\operatorname{vol}_{n-1}\left(U_{c}\right)$. Since the Gauss tangent degree is constant on $\widetilde{\mathcal{V}}$, this implies the continuity at $c$ of the function total curvature.

The converse of this result is not true as shown in the following example: take $f(x, y)=y\left(2 x^{2} y^{2}-9 x y+12\right)$ as a function on the real plane. Then we find that

$$
\lim _{t \rightarrow c^{-}}|K|(c)=\lim _{t \rightarrow c^{+}}|K|(c)=2 \pi, \text { while }|K|(0)=0,
$$

but $K$ is continuous at 0 .

Remark 6.7. Talking about the discontinuity of $|K|$ or $K$ makes only sense at values $c$ that are taken by the function $f$.

Let us return to the continuity of the curvature of $f$. Let $c$ be a regular value. For each $l=1, \ldots, N_{f}$, the Hausdorff $\operatorname{limit}$ of $\lim _{t \rightarrow c^{*}} \operatorname{clos}\left(\mathcal{U}_{l, c}\right)$ is denoted by $\mathscr{V}_{l}^{*}$, where $*=+$ or $*=-$.

Proposition 6.8. Let $c$ be a regular value at which $|K|$ is not continuous. There exists an open subset $U \subset \mathbb{S}^{n-1}$, such that for any $u \in U$, there exists a connected component $\Gamma$ of $\Gamma_{u}^{+}(f):=\Psi_{f}^{-1}(\{u\} \times \mathbb{R})$, such that $\Gamma \cap f^{-1}(c)$ is empty and one of the two cases below happens

(i) If $c$ is the infimum of $f$ along $\Gamma$, then for any $\varepsilon>0$ small enough, $\Gamma \cap f^{-1}(] c, c+\varepsilon[)$ is not bounded.

(ii) If $c$ is the supremum of $f$ along $\Gamma$, then for any $\varepsilon>0$ small enough, $\Gamma \cap f^{-1}(] c-\varepsilon, c[)$ is not bounded. 
Proof. Assume that $\lim _{t \rightarrow c^{-}}|K|(t)>|K|(c)$. The other situation is absolutely similar.

Let $\mathcal{U}_{c}=\sqcup_{i=1}^{d_{c}} U_{i}$, where $U_{i}$ is a connected component of $\mathcal{U}_{c}$. For each $i$, we have $U_{i} \subset \mathcal{U}_{s(i), c}$. Since $|K|(t)=\sum_{l=1}^{N_{f}} l \operatorname{vol}_{n-1}\left(\mathcal{U}_{l, t}\right)$, we thus find that $\lim _{t \rightarrow c^{-}}|K|(t)=\sum_{l=1}^{N_{f}} l \operatorname{vol}_{n-1}\left(\mathscr{V}_{l}^{-}\right)$.

For each $i=1, \ldots, d_{c}$, there exists a positive integer $l(i) \geqslant s(i)$ such that $\left.U_{i} \subset \mathscr{V}_{l(i)}^{-}\right)$. Thus the following is happening:

either

(a) there exists $i \in\left\{1, \ldots, d_{c}\right\}$ such that $l(i)>s(i)$

or

(b) there exists $i \in\left\{1, \ldots, d_{c}\right\}$ such that $\operatorname{vol}_{n-1}\left(\mathscr{V}_{l(i)}^{-}\right)>\operatorname{vol}_{n-1}\left(U_{i}\right)$.

Assume that a phenomenon of type (a) contributes to the discontinuity of $|K|$ at $c$. Then for each $u \in U_{i}$, there exists $\varepsilon>0$ such that $\Gamma_{u}^{+}(f) \cap f^{-1}(] c-$ $\varepsilon, c+\varepsilon[)$ has $m(i) \geqslant l(i)$, connected components $\mathcal{G}_{1}, \ldots, \mathcal{G}_{m(i)}$ such that, for $\left.j=1, \ldots, s(i), f\left(\mathcal{G}_{j}\right)=\right] c-\varepsilon, c+\varepsilon\left[\right.$, for $j=s(i)+1, \ldots, l(i), f\left(\mathcal{G}_{j}\right)=$ ]$c-\varepsilon, c\left[\right.$ and for $\left.j=l(i)+1, \ldots, m(i), f\left(\mathcal{G}_{j}\right)=\right] c, c+\varepsilon[$.

Let $j \in\{s(i)+1, \ldots, l(i)\}$. If $\mathcal{G}_{j}$ was bounded, its closure in $\mathbb{R}^{n}$ would then be $\operatorname{clos}\left(\mathcal{G}_{j}\right)=\mathcal{G}_{j} \cup\left\{x_{c-\varepsilon}, x_{c}\right\}$, where $x_{c-\varepsilon} \in F_{c-\varepsilon}$ and $x_{c} \in F_{c}$. Since $c$ is a regular value, this would mean that $\nabla f\left(x_{c}\right)=u\left|\nabla f\left(x_{c}\right)\right|$. Such a $u$ is a regular value of $\left.v_{f}\right|_{f^{-1}(] c-\varepsilon, c+\varepsilon[)}$, thus $\mathcal{G}_{j}$ could be extended to $\{f>c\}$ to a regular curve through $x_{c}$, which would contradict $\left.f\left(\mathcal{G}_{j}\right)=\right] c-\varepsilon, c[$. So we get that $\mathcal{G}_{j}$ never meet $f^{-1}(c)$. The same works for $j \in\{l(i)+1, \ldots, m(i)\}$. Case (b) is proved similarly.

There is a last thing to say about the case $\mathcal{U}_{c}$ is empty, meaning that $\mathrm{v}_{c}$ is of rank at most $n-2$. In this situation the discontinuity of $|K|$ is created by phenomenon of type (b) only.

When the discontinuity of $|K|$ arises at a critical value $c$, it is almost impossible to say anything similar to the previous statement, in the general frame we are given.

\section{Total $\lambda$-CURVATURE And total abSOlute $\lambda$-CURVATURE}

Given a compact connected manifold $M$ of dimension $m$, given a Morse function $g: M \mapsto \mathbb{R}$, let $C_{\lambda}(g)$ be the set of critical points of index $\lambda$. The weak Morse inequalities state

$$
\# C_{\lambda}(g) \geqslant b_{\lambda}(M), \text { the } \lambda \text {-th Betti number. }
$$

Let us consider now that $M$ is an orientable connected definable $C^{l}, l \geqslant 2$, hypersurface of $\mathbb{R}^{n}$, with orientation map $v_{M}$.

Using Definition 3.4 given $\lambda \in\{0, \ldots, n-1\}$, let us define 


$$
I_{M}(\lambda):=\left\{x \in M: \lambda_{M}(x)=\lambda\right\} .
$$

Since $I_{M}(\lambda) \subset M \backslash \operatorname{crit}\left(\vee_{M}\right)$, and $M \backslash \operatorname{crit}\left(\vee_{M}\right) \ni x \mapsto \lambda_{M}(x)$ is definable and locally constant, the subset $I_{M}(\lambda)$ is definable.

Now we can define

Definition 7.1. Given $\lambda \in\{0, \ldots, n-1\}$,

(1) the total $\lambda$-curvature of $M$ is $K_{M}(\lambda):=\int_{I_{M}(\lambda)} k_{M}(x) \mathrm{d} v(x)$,

(2) the total absolute $\lambda$-curvature of $M$ is $|K|_{M}(\lambda):=\int_{I_{M}(\lambda)}\left|k_{M}\right|(x) \mathrm{d} v(x)$.

If $M$ is compact then averaging on the restriction to $M$ of all the oriented linear projections we deduce

$$
|K|_{M}(\lambda) \geqslant \operatorname{vol}_{n-1}\left(\mathrm{v}_{M}\left(I_{M}(\lambda)\right)\right) \cdot b_{\lambda}(M) .
$$

Now let us come to the case of definable functions. Let $f: \mathbb{R}^{n} \mapsto \mathbb{R}$ still be a $C^{l}$, definable function with $l \geqslant 2$. We use the notations of Section 4 and Section 5. Given any $t \notin K_{0}(f)$, let $\mathrm{v}_{t}$ be the restriction of $\mathrm{v}_{f}$ to $F_{t}$, and let $k_{t}(x)$ be the Gauss curvature at $x \in F_{t}$.

Let us define the open definable subset

$$
I_{f}(\lambda):=\left\{x \notin \operatorname{crit}(f) \cup \operatorname{crit}\left(v_{f}\right): \text { the index of }\left.\mathrm{d}_{x} v_{f}\right|_{T_{x} F_{f^{-1}(x)}} \text { is } \lambda\right\}
$$

Let $\mathcal{E}_{t}$ still be the set of connected components of $F_{t}$. Given $t \notin K_{0}(f)$ and $\lambda \in\{0, \ldots, n-1\}$, let

$$
I_{t}(\lambda)=\cup_{E \in \mathcal{E}_{t}} I_{E}(\lambda)=I_{f}(\lambda) \cap F_{t} .
$$

The family $\left(\mathrm{v}_{t}\left(I_{t}(\lambda)\right)_{t \notin K_{0}(f)}\right)$ is a definable family of subsets of $\mathbb{S}^{n-1}$.

We define two new functions of $t$, the total $\lambda$-curvature of $f$ :

$$
K(\lambda ; t):=\sum_{E \in \mathcal{E}_{t}} K_{E}(\lambda)=\int_{I_{t}(\lambda)} k_{t}(x) \mathrm{d} v(x)
$$

and the total absolute $\lambda$-curvature of $f$ :

$$
|K|(\lambda ; t):=\sum_{E \in \mathcal{E}_{t}}|K|_{E}(\lambda)=\int_{I_{t}(\lambda)}\left|k_{t}\right|(x) \mathrm{d} v(x) .
$$

Now we can state the main result of this section

Theorem 7.2. Let $\lambda \in\{1, \ldots, n-1\}$ be given.

(1) Let $c$ be a value. Then $\lim _{t \rightarrow c^{-}} K(\lambda ; t), \lim _{t \rightarrow c^{+}} K(\lambda ; t), \lim _{t \rightarrow c^{-}}|K|(\lambda ; t)$ and $\lim _{t \rightarrow c^{+}}|K|(\lambda ; t)$ exist.

(2) Let $c$ be a value. Then

$$
|K|(\lambda ; t) \leqslant \min \left\{\lim _{t \rightarrow c^{-}}|K|(\lambda ; t), \lim _{t \rightarrow c^{+}}|K|(\lambda ; t)\right\} .
$$


(3) The function $t \rightarrow|K|(\lambda ; t)$ admits at most finitely many discontinuities.

(4) If the function $t \rightarrow|K|(\lambda ; t)$ is continuous at $c$, then so is the function $t \rightarrow K(\lambda ; t)$.

Proof. The proof of each point works as the proof of the similar statement given for the functions $t \rightarrow K(t)$ and $t \rightarrow|K|(t)$. The reason for that is to consider the function $\Psi_{\lambda}:=\left.\Psi_{f}\right|_{I_{f}(\lambda)}$, and then do exactly the same work as that done in Section 5 and 6

\section{REMARKS, COMMENTS AND SUGGESTIONS}

In the introduction we mentioned that the original motivation was to try to find some equisingularity conditions on the family of levels $\left(F_{t}\right)$ that could be read through the continuity of these total curvature functions. We were especially interested in the problem caused by the regular values that are also bifurcation values. This phenomenon is without any doubt caused by some curvature, in the broader sense of Lipschitz-Killing curvature, accumulation at infinity (i.e. on the boundary of the domain). Without any bound on the complexity of the singularity phenomenon occurring at infinity, it would be very naive and wrong to hope that the continuity of these curvature functions we dealt with is a sufficiently fine measure of the equisingularity of the levels $F_{t}$ nearby a regular value. In some simple cases, see [Gr], they can provide sufficient conditions to ensure the equisingularity in a neighborhood of a given regular asymptotic critical value. But in whole generality we may also have to consider these higher order curvatures.

Consider again the situation of Section 4: That is of $f: \mathbb{R}^{n} \mapsto \mathbb{R}$ a definable function enough differentiable. We have associated to each regular value $t$ of $f$, two real numbers, namely $K(t)$ and $|K|(t)$.

Let $t$ be a regular value of $f$ and let $x \in F_{t}$. Let $q \in\{1, \ldots, n-1\}$ be a given integer. Let $N$ be a $q$-dimensional sub-vector space of $T_{x} F_{t}$. Let us denotes $k_{t}(N, x)$ the Gauss curvature at $x$ of $F_{t} \cap\left(x+\left(\mathbb{R} v_{t}(x) \oplus N\right)\right)$ a hypersurface of the $q+1$-dimensional affine subspace $x+\left(\mathbb{R} v_{t}(x) \oplus N\right)$. The $q$-th Lipschitz-Killing curvature of $F_{t}$ at $x$ is the real number

$$
L K_{q}(x):=\int_{G\left(q, T_{x} F_{t}\right)} k_{t}(N, x) \mathrm{d} N
$$

where $\mathrm{d} N$ is the volume form of $G\left(q, T_{x} F_{t}\right)$ the Grassmann manifold of $q$ dimensional sub-vector spaces of $T_{x} F_{t}$.

So we would like to define two real numbers:

the total $q$-th Lipschitz-Killing curvature, 


$$
L_{q}(t)=c_{n, q} \int_{F_{t}} L K_{q}(x) \mathrm{d} v_{n-1}(x),
$$

and the total absolute $q$-th Lipschitz-Killing curvature, also called total $q$-th length

$$
|L|_{q}(t)=c_{n, q} \int_{F_{t}}\left|L K_{q}(x)\right| \mathrm{d} v_{n-1}(x),
$$

where $c_{n, q}$ is a universal constant depending only on $q$ and $n$.

In general, once $q<n-1$, with the level $F_{t}$ non compact, the number $|L|_{q}(t)$ is likely to be infinite !

A more relevant question would be to estimate the first (and may be the second) dominant term of the asymptotic of

$$
\begin{aligned}
|L|_{q}(t ; R) & =c_{n, q} \int_{F_{t} \cap \mathbb{B}_{R}^{n}}\left|L K_{q}(x)\right| \mathrm{d} v_{n-1}(x) \\
L_{q}(t ; R) & =c_{n, q} \int_{F_{t} \cap \mathbb{B}_{R}^{n}} L K_{q}(x) \mathrm{d} v_{n-1}(x)
\end{aligned}
$$

as $R \rightarrow+\infty$ and to see how these dominant terms varies in $t$ or in $(t, R)$. But as already said, even in the subanalytic category where results from $[\mathrm{CLR},[\mathrm{LR}]$ provide some general information on the nature of such terms, it is likely to be a very difficult question !

\section{REFERENCES}

[BB] A. Bernig and L. Bröcker, Courbures intrinsèques dans les catégories analyticogéométriques, Ann. Inst. Fourier (Grenoble) 53 (2003), no. 6, 1897-1924.

[Br] L. Bröcker, Families of semialgebraic sets and limits, in Real algebraic geometry (Rennes, 1991), 145-162, Lecture Notes in Math., 1524, Springer, Berlin.

[BK] L. Bröcker \& M. Kuppe, Integral Geometry of Tame Sets, Geom. Dedicata, 82 (2000) 285-323.

[Com] G. Comte, Équisingularité réelle: nombres de Lelong et images polaires, Ann. Sci. École Norm. Sup. (4) 33 (2000), no. 6, 757-788.

[Cos] M. Coste, An Introduction to O-minimal Geometry, Istituti Editoriali e Poligrafici Internazionali, Pisa (2000), 82 pages, also available on http://perso.univ-rennes1.fr/michel.coste/polyens/OMIN.pdf

[CLR] G. Comte \& J.-M.Lion \& J.-P. Rolin, Nature log-analytique du volume des sousanalytiques, Ill. J. Math. 44 (2000), No.4, 884-888.

[vD1] L. van den Dries, Tame topology and o-minimal structures, Cambridge University Press, Cambridge, 1998.

[vD2] L. van dan Dries, Limit sets in o-minimal structure, in Proceedings of the RAAG Summer School Lisbon 2003: O-minimal Structures, (2005), 172-214, available on http://www.uni-regensburg.de/Fakultaeten/nat_Fak_I/RAAG/preprints/0159.html

[vDM] L. van den Dries \& C. Miller, Geometric Categories and o-minimal strucutures, Duke Math. J., 84 (1996) 497-540. 
[Fu] J. Fu, Curvature Measures of Subanalytic Sets, American J. Math., 116 (1994) 819-880.

[Gr] V. Grandjean, Tame Functions with strongly isolated singularities at infinity: a tame version of a Parusinski's Theorem, to appear in Geometriae Dedicata, preprint available on http://arxiv.org/PS_cache/arxiv/pdf/0708/0708.2474v3.pdf

[Ka] T. Kaiser, On convergence of integrals in o-minimal structures on archimedean real closed fields, Ann. Pol. Math. 87, (2005) 175-192.

[Le] O. Le Gal, Modèle complétude des structures o-minimales polynomialement bornées) Thèse de doctorat, Université de Rennes I (2006).

[LR] J.M. Lion \& J.P. Rolin, Volumes, feuilles de Rolle de feuilletages analytiques et théorème de Wilkie, Ann. Fac. Sci. Toulouse Math. (6) 7 (1998), no. 1, 93-112.

[LS] J.M. Lion \& P. Speissegger, A geometric proof of the definability of Hausdorff Limits, Sel. Math., New Ser. 10 (2004), no. 3, 377-390.

Permanent Address: V. GRandjean, Department of Computer Science, UniVERSITY OF BATH, BATH BA2 7AY, ENGLAND,(UNITED KINGDOM)

Current Address: V. GRandJean, FAKUlät V, Institut FÜr Mathematik CARL VON OSSIETZKY UNIVERSITÄT, OLDENBURG, 26111 OLDENBURG I.O. (GERMANY)

E-mail address: cssvg@bath.ac.uk 